\title{
Optimal design of gear ratio using non-circular gear for jumping robot
}

\author{
Masafumi OKADA* and Yushi TAKEDA* \\ * Dept. of Mechanical Sciences and Engineering, Tokyo Institute of Technology \\ 2-12-1 Oookayama Meguro-ku Tokyo 152-8552, JAPAN \\ E-mail: okada@mep.titech.ac.jp
}

Received 4 December 2014

\begin{abstract}
For the effective use of motor power, an appropriate gear ratio has to be selected according to robot tasks and motions. Because a jumping robot, in particular, requires both high torque and high velocity properties through its motion, a varying gear ratio will realize a high performance. Moreover, its varying property has to be optimally designed and realized. In this paper, we design a jumping robot with a non-circular gear which changes the gear ratio through the motion for the higher jumping. The gear ratio is optimized so that the motor power is maximized, and the change of the gear ratio is obtained based on a forward dynamical analysis. The optimized gear ratio is realized by a non-circular gear considering a constraint of pressure angle of gear teeth. A jumping robot is prototyped, and the effectiveness of the proposed design method is verified considering model perturbations of the physical parameters of the robot.
\end{abstract}

Key words : Varying gear ratio, Non-circular gear, Jumping robot

\section{Introduction}

For mechanical design of a robot system, a reduction gear is utilized to change the actuator property. Supposing the power of a DC (Direct Current) motor is $P[\mathrm{~W}]$, the motor torque $\tau[\mathrm{Nm}]$ and its angular velocity $\omega[\mathrm{rad} / \mathrm{sec}] \mathrm{have}$ a relationship of $P=\tau \omega$. The limitation of $\tau$ and $\omega$ is decided by the motor current and input voltage respectively, and these limitations are decided by a power supply or a motor driver. Therefore, an appropriate gear ratio has to be selected for the effective use of motor power. Assuming the gear ratio as $G, \tau$ is multiplied by $1 / G$ and $\omega$ is multiplied by $G$. When a large torque is required, a small $G$ is selected by the sacrifice of small angular velocity. In general, because $G$ is a constant value, an appropriate $G$ is selected according to the robot motion and task. On the other hand, many tasks require both large torque output and high velocity realization. For example, to carry a heavy weight, large torque is required to lift up and high velocity to convey it. For these tasks, a varying gear ratio is effective for continuous motions without gear change.

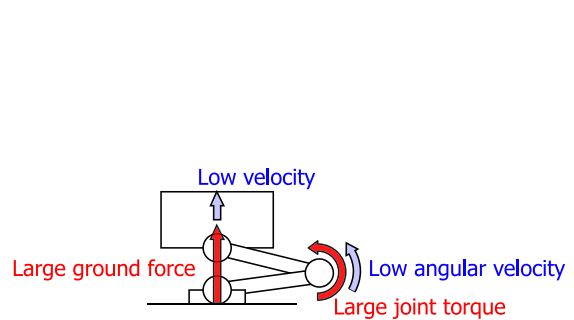

(a) Initial position

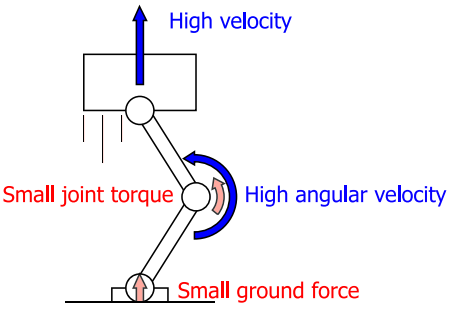

(b) Final position

Fig. 1 Requirements for jumping motion

As a typical case of requirement for varying property of large torque output and high velocity realization through the motion, we consider a jumping robot. As shown in Fig. 1, a jumping robot requires (a) a large torque output to 
kick the ground in the beginning of the motion, and (b) high velocity realization in the end of the motion. For the better performance, i.e. the higher jumping, a continuously varying gear ratio through the motion is necessary.

Many researches on a jumping robot have been reported so far. Niiyama (Niiyama and Kuniyoshi, 2009) considered humanoid running with a musculoskeletal mechanism. They proposed convex polygon of forces at a foot link, and investigated the landing property depending on the force controller on each joint. Ishikawa (Ishikawa, Neki, Imura and Hara, 2003) proposed a motion stabilization method of a hopping robot based on a hybrid-system control theory. Ugurlu (Ugurlu and Kawamura, 2009) designed a ZMP-based jumping controller. These methods focused on a control of stable jumping. Shimoda (Shimoda, Kubota and Nakatani, 2002) and Sakaguchi (Sakaguchi, Sudo, Bushida, Chiba, Asai and Kikuchi, 2007) developed jumping principal and mechanism using inertia force of a mass inside the body. Kovac (Kovac, Fuchs, Guignard, Zufferey and Floreano, 2008), Curran (Curran and Orin, 2008) and Tsuda (Tsuda, Mochiyama and Fujimoto, 2009) proposed a jumping mechanism using a spring. In these mechanisms, the robot realizes the higher jumping by using the accumulated energy of springs or inertia forces, however, it may consume large energy of the actuator because not all energy is available for jumping.

In this paper, we develop a design method of the optimal varying gear ratio for a jumping motion aiming at the effective use of actuators, and the obtained gear ratio is realized by a non-circular gear. A design method of the pitch curve of the non-circular gear is proposed, and the effectiveness of the non-circular gear is evaluated by a prototype. Moreover, because the pitch curve is designed based on a simulation-based method, namely, forward dynamical analysis, it is effective only for a specified robot with a specified initial posture. In this paper, we also investigate the change of the jumping height with respect to the perturbation of a robot parameter and initial posture.

CVT (Continuously Variable Transmission) is used for an automobile transmission that realizes a varying gear ratio, however, it is for a multiple rotational actuator with high angular velocity. Hagiwara (Hagiwara and Hirose, 1999) and Takaki (Takaki, Sugiyama, Takayama and Omata, 2006) proposed special mechanisms that realize load sensitive varying gear ratio. The proposed method in this paper focuses on a fixed but position depending gear ratio for a robot motion.

\section{Jumping robot and optimal gear ratio}

\subsection{Optimization of gear ratio}

In this section, we derive an optimal gear ratio that maximizes a ground force. Consider a jumping robot shown in Fig. 2. As shown in Fig. 2(a), a DC motor is mounted on the body. $\phi_{m}$ and $\tau_{m}$ represent a rotational angle and output

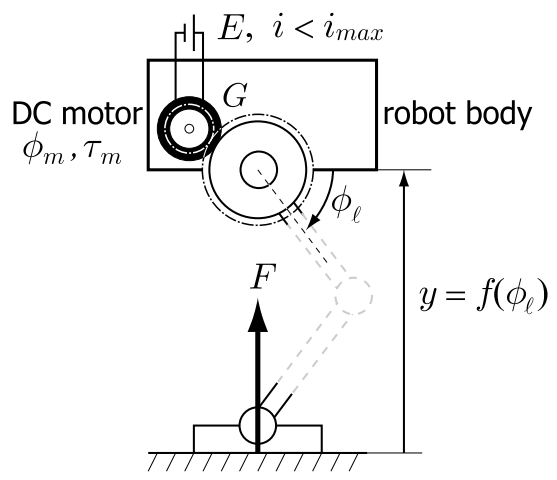

(a) Mechanism of a jumping robot

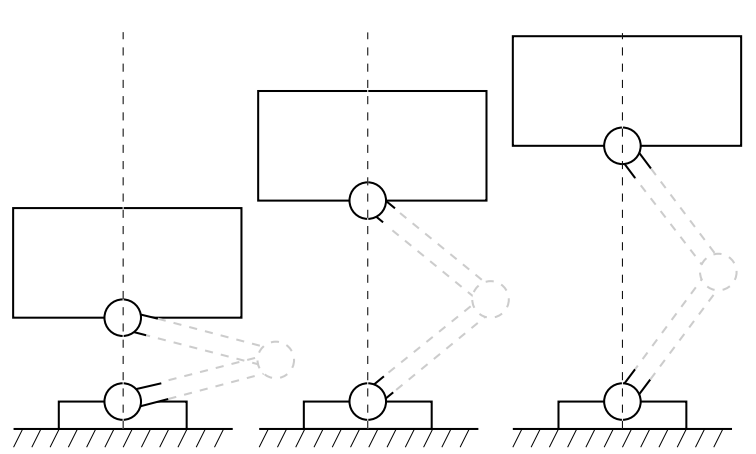

(b) Motion of the jumping robot

Fig. 2 Jumping robot and its motion

torque of the motor, respectively. A constant voltage $E$ is supplied to the motor, and the maximum value of the motor current $i$ is limited by $i_{\text {max }} . \phi_{m}$ is transferred to the rotation of a leg link $\phi_{\ell}$ with a gear whose gear ratio is $G$, which means

$$
\dot{\phi}_{\ell}=G \dot{\phi}_{m}
$$

is satisfied. The configuration of the leg mechanism is out of consideration in this section, but its kinematic relationship between $\phi_{\ell}$ and the height of the body $y$ and their velocity are assumed to be

$$
y=f\left(\phi_{\ell}\right), \quad \dot{y}=J\left(\phi_{\ell}\right) \dot{\phi}_{\ell}, \quad J=\frac{\partial f}{\partial \phi_{\ell}}
$$

where $J$ represents Jacobian. Moreover, because of some kinematic constrains, the motion of the robot body is assumed to be constrained in the vertical line that crosses the centers of ankle and hip joints as shown in Fig. 2 (b), which means this robot has one degree-of-freedom. 
The motor torque $\tau_{m}$ yields vertical ground force $F$, and we consider the maximization of $F$ designing an optimal gear ratio. The kinetic energy $e$ of the robot is represented by;

$$
e=\int_{0}^{y_{o f f}} F d y
$$

where $y_{\text {off }}$ means the body height at taking off. Because robot takes off when $F<0$ is satisfied, the larger $F$ takes the longer time of $F \geq 0$ and the higher jumping.

From the relationship of voltage between the motor terminals, the following equation is derived;

$$
E=i R+L \frac{d i}{d t}+K_{a} \dot{\phi}_{m}
$$

where $R$ means a motor resistance, $L$ means a motor inductance and $K_{a}$ is a torque constant ( = back electromotive force constant). By assuming $L$ is negligible small, $i$ is represented by;

$$
i=\frac{1}{R}\left(E-K_{a} \dot{\phi}_{m}\right)
$$

and $\tau_{m}$ is represented by:

$$
\tau_{m}=K_{a} i=\frac{K_{a}}{R}\left(E-K_{a} \dot{\phi}_{m}\right)
$$

On the other hand, from Eqs. (1), (2) and virtual work principle, $F$ is represented by:

$$
F=(G J)^{-1} \tau_{m}
$$

Moreover, substituting Eqs. (2) and (6) into (7),

$$
F=\frac{G^{-1}}{J} \frac{K_{a}}{R}\left(E-K_{a} \frac{G^{-1}}{J} \dot{y}\right)
$$

is obtained. Because $J>0$ and $\dot{y}>0$ are satisfied without loss of generality, Eq. (8) is convex upward with respect to $G^{-1}$, and the optimal $G$ that maximizes $F$ is obtained by:

$$
G=\frac{2 K_{a} \dot{y}}{E J}
$$

On the other hand, the motor power $P$ is represented by:

$$
P=\tau_{m} \dot{\phi}_{m}=F \dot{y}
$$

From Eqs. (8) and (10), the optimal gear ratio in Eq. (9) also maximizes $P$.

In Eq. (9), $K_{a}$ and $E$ are constant, and $J$ is a function of $\phi_{m}$. However, because $\dot{y}$ represents a velocity, $G$ is a function of velocity which is difficult to be realized by a mechanism. In this paper, the relationship between $\dot{y}$ and $\phi_{m}$ is obtained using forward dynamical analysis, and $G$ is realized by a non-circular gear.

\subsection{Shape design of non-circular gear based on forward dynamical analysis}

In this section, the relationship between $\dot{y}$ and $\phi_{m}$ is obtained based on forward dynamical analysis. The procedure is as follows.

Step 1 The robot dynamics is represented by;

$$
M_{m}\left(\phi_{m}\right) \ddot{\phi}_{m}+C_{m}\left(\phi_{m}, \dot{\phi}_{m}\right)+K_{m}\left(\phi_{m}\right)=\tau_{m}
$$

where $\phi_{m}$ is a generalized coordinates, $M_{m}$ is an inertia, $C_{m}$ is a Coriori and centrifigal term, $K_{m}$ is a gravity term. Set the initial value $\phi_{m}(0)=\phi_{m 0}, \dot{\phi}_{m}(0)=0$ at $t=0$.

Step 2 From $\dot{\phi}_{m}, J$ and Eq. (1), $\dot{y}$ is obtained and the optimal gear ratio $G$ is calculated from Eq. (9). Because extremely small $G$ cannot be realized by a non-circular gear, the minimum value of $G$ is limited by $G_{\min }$ as:

$$
G=\left\{\begin{array}{l}
G_{\min }\left(\frac{2 K_{a} \dot{y}}{E J}<G_{\min }\right) \\
\frac{2 K_{a} \dot{y}}{E J}\left(\frac{2 K_{a} \dot{y}}{E J} \geq G_{\min }\right)
\end{array}\right.
$$




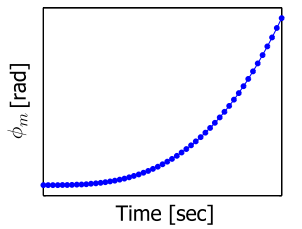

(a) time variation of $\phi_{m}$ and $G$

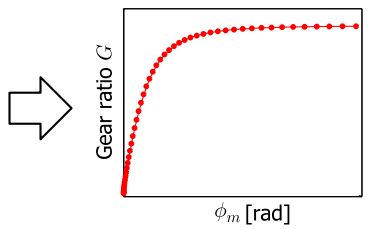

(b) relationship bitween $\phi_{m}$ and $G$

Fig. 3 Obtained $\phi_{m}$ and $G$

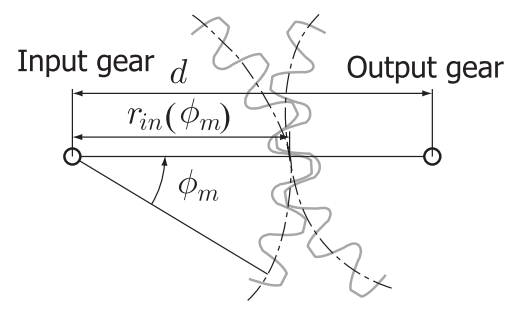

Fig. 4 Radius of input gear

Step $3 \quad \ddot{\phi}_{m}$ is calculated from Eq. (11) using $G$ in Step 2, and $\dot{\phi}_{m}$ and $\phi_{m}$ in the next step are calculated by integrating $\ddot{\phi}_{m}$ and $\dot{\phi}_{m}$. Moreover, from the time differential of Eq. (1), the following equation is obtained;

$$
\ddot{\phi}_{\ell}=G \ddot{\phi}_{m}+\frac{\partial G}{\partial \phi_{m}} \dot{\phi}_{m}^{2}
$$

and $\phi_{\ell}$ is calculated by 2 nd order integral of Eq. (13). $\partial G / \partial \phi_{m}$ is approximated by;

$$
\frac{\partial G}{\partial \phi_{m}} \simeq \frac{\Delta G}{\Delta \phi_{m}}
$$

where $\Delta G$ and $\Delta \phi_{m}$ represent the difference of $G$ and $\phi_{m}$ between the current and previous iteration values.

Step 4 From Eq. (8), $F$ is calculated using Eq. (2). When $F>0$, go to Step 2 and iterate the same procedure, or when $F \leq 0$, stop the iteration.

By this procedure, time variations of $\phi_{m}$ and $G$ are obtained as shown in Fig. 3 (a), and we obtain the relationship between $\phi_{m}$ and $G$ as $G=G\left(\phi_{m}\right)$ as shown in Fig. 3 (b). By defining the distance between the centers of input and output non-circular gear as $d$ as shown in Fig. 4, the pitch radius of the input gear $r_{\text {in }}$ is obtained by:

$$
r_{i n}\left(\phi_{m}\right)=\frac{G\left(\phi_{m}\right) d}{1+G\left(\phi_{m}\right)}
$$

The pitch radius of the output gear $r_{\text {out }}$ is also obtained by the same way.

\subsection{Discussions on the proposed method}

(1) Because the optimal gear ratio is obtained based on the robot dynamics, $G$ is effective only for a specified robot.

(2) Because the change of $G$ is obtained from the forward dynamical analysis with an initial value, it is effective only for a specified initial position of the jumping robot.

(3) The initial value of $\dot{\phi}_{m}$ is set as zero, which causes $\dot{y}(0)=0$ and $G=0$. However, zero gear ratio cannot be realized by a non-circular gear. Moreover it will be changed drastically to large according to the motion, which causes large pressure angle of the gear tooth. It is known that the pressure angle has to be less than $50^{\circ}$ (Katori, 2001). To overcome this problem, an appropriate $G_{\min }$ has to be selected.

(4) Because Eq. (9) contains Jacobian $J$ which is the information of leg mechanism, $G$ is optimized for any leg mechanisms. It is true that an appropriate design of leg mechanism i.e. appropriate $J$ will realize maximization of the ground force, however, the nonlinearity of the mechanism has limitations. On the other hand, a non-circular gear realizes almost arbitrary function, and it complements the limitation of the mechanism.

\section{Design of jumping robot and non-circular gear}

\subsection{Configuration of jumping robot}

In this section, a non-circular gear is designed for a jumping robot. Figure 5 shows a configuration of the jumping robot. As shown in Fig. 5 (a), the rotation of a motor is transmitted to the rotation of the leg through Gear 1, 2 and 3 whose gear ratio are $G_{1}, G_{2}$ and $G_{3}$ respectively. $G_{1}$ is small to increase the torque, $G_{2}$ is a varying gear ratio realized by a non-circular gear and $G_{3}$ is more than 1 to broaden the effective area of the non-circular gear because the leg rotates less than $90^{\circ}$. For this robot, $G$ in Fig. 2 (a) is represented by; $G=G_{1} G_{2} G_{3}$. the motion of the body is restricted to the vertical direction by a linear slider. The upper leg and lower leg are connected by a timing pulley as shown in Fig. 5 (b). The gear ratio of the timing pulley is 2 that realizes the motion shown in Fig. 2 (b). By setting the length of the upper and lower legs as $\ell, J$ in Eq. (2) is represented by;

$$
J=2 \ell \cos \left(\phi_{\ell}+\phi_{\ell 0}\right)
$$




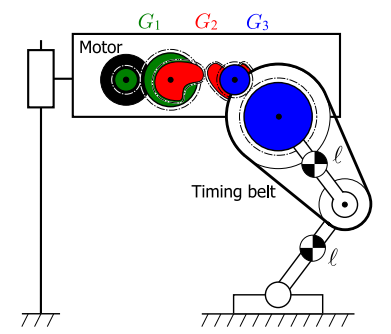

(a) configuration of three gears

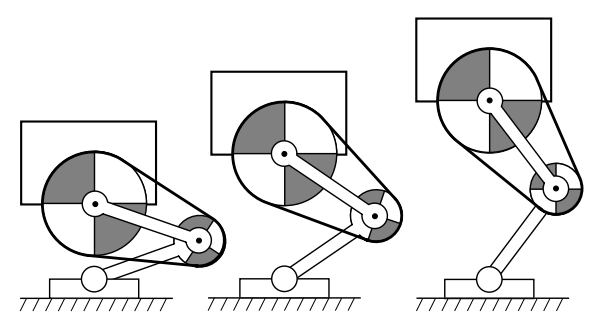

(b) motion of the robot

Fig. 5 Jumping robot and its mechanism

and the optimal gear ratio $G_{2}$ is given by;

$$
G_{2}=\frac{K_{a} \dot{y}}{G_{1} G_{3} E \ell \cos \left(\phi_{\ell}+\phi_{\ell 0}\right)}
$$

where $\phi_{\ell 0}$ means the initial value of $\phi_{\ell}$. The body weight of the robot is $1.65 \mathrm{~kg}, \ell$ is $0.15 \mathrm{~m}$, and $G_{1}=1 / 28, G_{3}=1 / 5$ are set. The friction term of Gear 1 and the linear slide are considered which are measured from the experimental system in section 4. 90W-DC motor (MAXON Corp.) is utilized, whose resistance $R=2.07 \Omega$, torque constant $K_{a}=$ $52.5 \times 10^{-3} \mathrm{Nm} / \mathrm{A}$. $E$ is set as $48 \mathrm{~V}$ and current limitation $i_{\max }=12 \mathrm{~A}$ which is the limitation of a motor driver. $\phi_{\ell 0}=10^{\circ}$ is set.

\subsection{Design of non-circular gear}

Based on the proposed algorithm, a non-circular gear is designed. Figure 6 shows the result of a forward dynamical simulation using $G_{2 \min }=0.1$. Figure 6 (a), (b), (c) and (d) represent time variation of gear ratio $G_{2}$, body velocity $\dot{y}$, ground force $F$ and motor current $i$, respectively.

(1) From Fig. 6 (c), the ground force is zero at $t=0.221 \mathrm{sec}$, which means the robot takes off at this time.

( 2 ) From Fig. 6 (b), the body velocity at taking off is $1.69 \mathrm{~m} / \mathrm{sec}$, which yields the jumping height of $0.124 \mathrm{~m}$, where the jumping height is defined by Fig. 7.

( 3 ) In Fig. 6 (a), the gear ratio is restricted by $G_{2 \min }$ in the beginning of the motion. After $t=0.051, G_{2}$ changes larger. However, its change is so large that the changes of body velocity and ground force are also too large. Moreover it yields large pressure angle of the gear tooth.

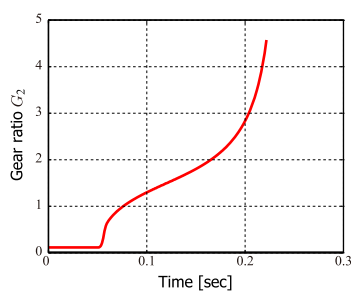

(a) gear ratio

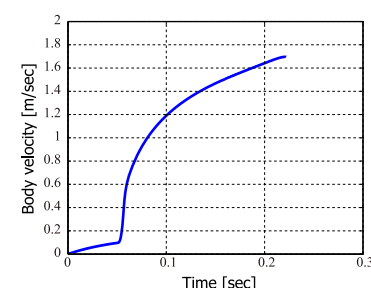

(b) body velocity

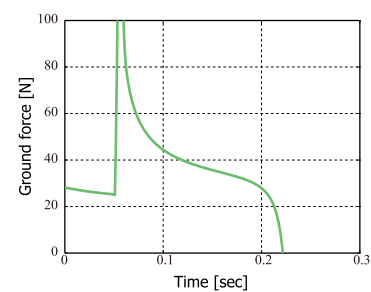

(c) ground force

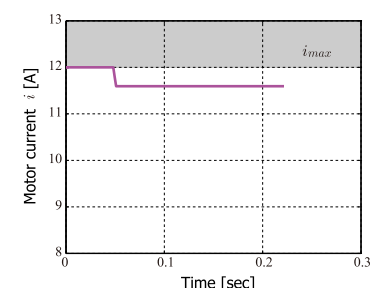

(d) motor current

Fig. 6 Simulation results of the jumping motion with $G_{2 \min }=0.1$

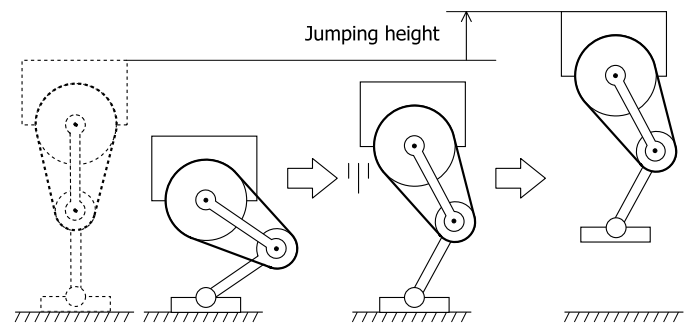

Fig. 7 Definition of jumping height

(4) In Fig. 6 (d), the motor current is restricted by $i_{\max }$ in the beginning of the motion. After $t=0.051$, it converges to

$$
i=\frac{E}{2 R}
$$

which is introduced from Eq. (5) using $\dot{y}=J \dot{\phi}_{\ell}$, Eq. (1) and (9). 


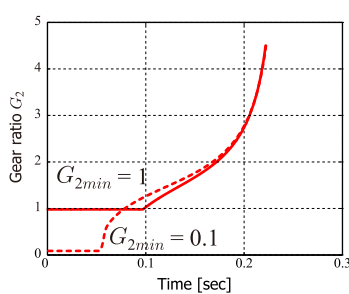

(a) gear ratio

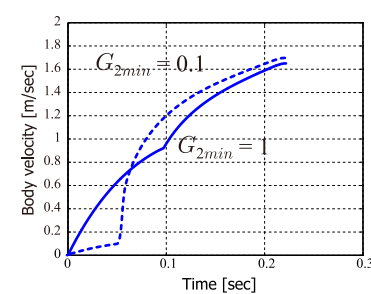

(b) body velocity

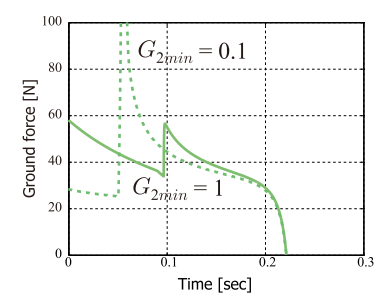

(c) ground force

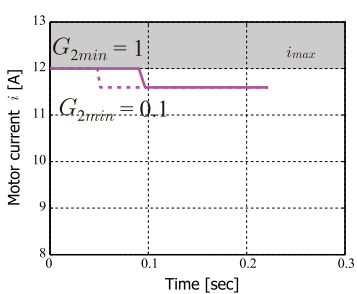

(d) motor current

Fig. 8 Simulation results of the jumping motion with $G_{2 \min }=1$

From the restriction of the pressure angle of gear tooth, the obtained gear ratio cannot be realized by a non-circular gear. This is because $G_{2 \min }=0.1$ is too small. And we redesign $G_{2}$ by setting $G_{2 \min }=1$, and results are shown in Fig. 8 by solid lines. The results in Fig. 7 are shown by dashed lines for comparison. In this result, the robot takes off at $t=0.221$ with the body velocity $1.64 \mathrm{~m} / \mathrm{sec}$, which yields $0.117 \mathrm{~m}$ of jumping height (only $5.6 \%$ lower than $G_{2 \mathrm{~min}}=0.1$ ). The shapes of the non-circular gears (pitch curve) are shown in Fig. 9 and the pressure angles of gear tooth are shown in Fig.10. The pressure angle is defined by $\alpha$ in Fig. 11. The distance between the center of the input and output gear is

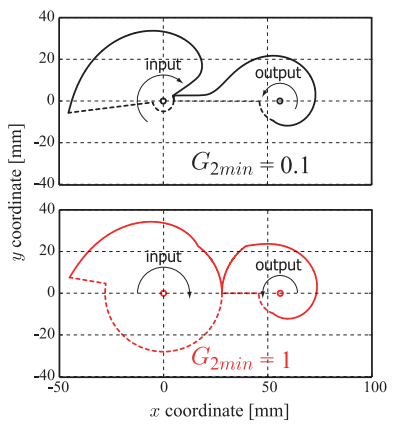

Fig. 9 Pitch curve of the non-circular gear

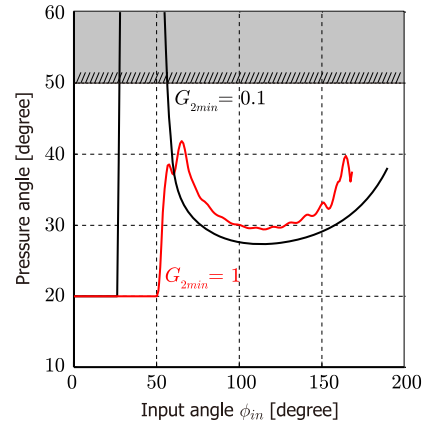

Fig. 10 Pressure angle of gear tooth

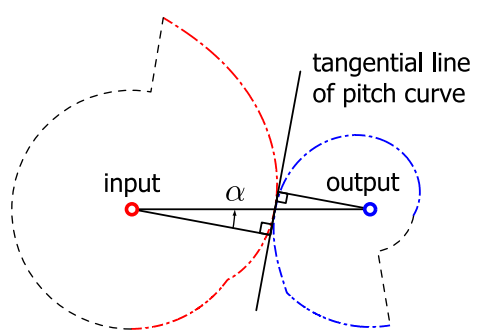

Fig. 11 Definition of pressure angle

$0.056 \mathrm{~m}$. Because the dashed lines in Fig. 9 are not used, the shape is arbitrary. From Fig. 10, by setting $G_{2 m i n}=1$, the pressure angle of the gear tooth changed less than $50^{\circ}$.

\subsection{Comparison with circular gear}

To show the effectiveness of the non-circular gear, the optimal constant gear ratio using circular gear is calculated. Figure 12 shows the relationship between constant gear ratio and jumping height. $G_{2}$ is set as a constant value and the

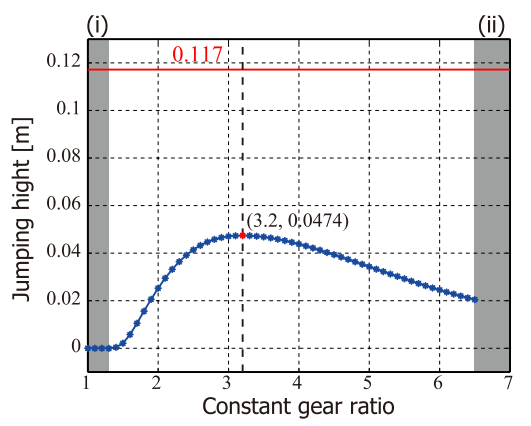

Fig. 12 The relationship between constant gear ratio and jumping height

jumping height is calculated by simulations. In the gray area (i), the robot dose not jump because the gear ratio is small (body velocity is small). In the area (ii), the robot does not stand up because of small torque with high gear ratio. From this result, the robot jumps highest $(0.0474 \mathrm{~m})$ by setting $G_{2}=3.2$, however, it is much smaller than by using non-circular gear $\left(0.117 \mathrm{~m}, G_{2 \min }=1\right)$.

The simulation results using $G_{2}=3.2$ are shown in Fig. 13. The results in Fig. $8\left(G_{2 m i n}=1\right)$ are shown by dashed lines. The robot takes off at $t=0.275$. Figure 13 (d) shows the circular gear uses maximum motor current $(=12 \mathrm{~A})$ i.e. maximum motor torque through the motion. However, the jumping height is only $0.0474 \mathrm{~m}$ which is $41 \%$ of non-circular gear. To investigate this reason, motor power and work are calculated. The results are shown in Fig. 14 and 15 . The motor 


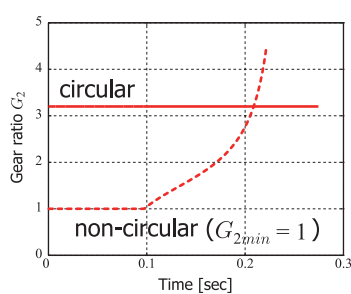

(a) gear ratio

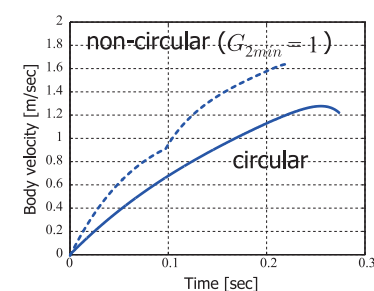

(b) body velocity

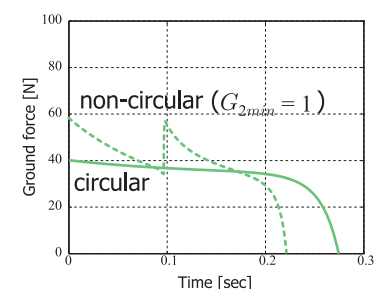

(c) ground force

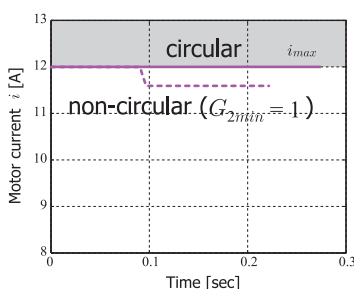

(d) motor current

Fig. 13 Simulation results of jumping motion using constant gear ratio $\left(G_{2}=3.2\right)$

power is calculated by $P=\tau_{m} \dot{\phi}_{m}$ and the motor work $W$ is time integral of $P$. From these results, we can see that by

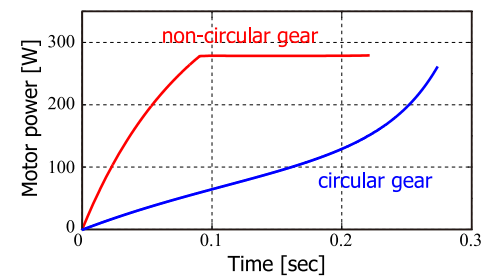

Fig. 14 Motor power through the motion

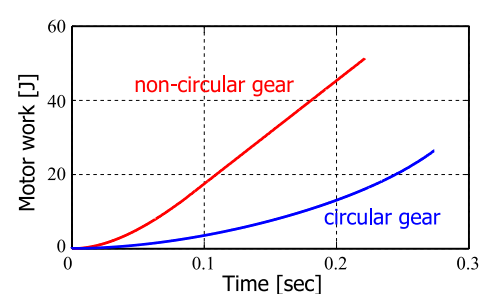

Fig. 15 Motor work through the motion

using the non-circular gear, motor power is much larger than by using circular gear, and it is concluded that the proposed method is effective use of motor power. The motion of the robot is shown in Fig. 16. The red line uses the non-circular

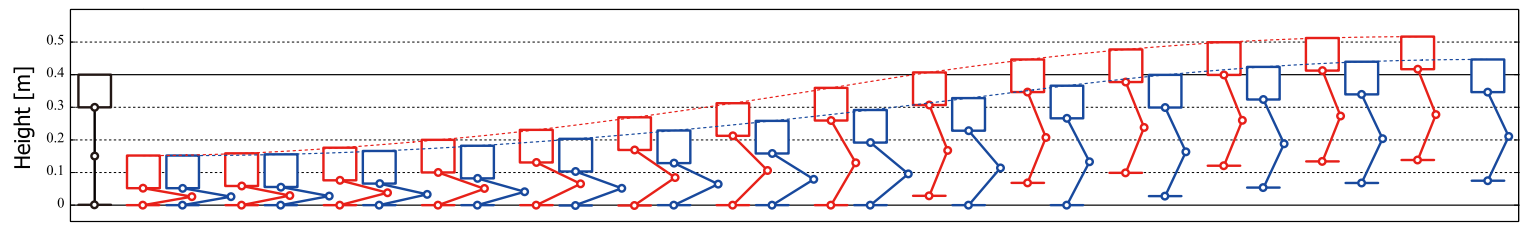

Fig. 16 Jumping motion (simulation results)

gear and the blue line uses the circular gear.

\subsection{Sensitivity of jumping height to parameter perturbation}

The non-circular gear in Fig. 9 is designed for a specified robot and specified initial position based on forward dynamical analysis. It is necessary to evaluate the change of the jumping height with respect to a parameter perturbation. The non-circular gear is designed using the body weight $M=1.65 \mathrm{~kg}$ and the initial angle of leg $\phi_{\ell 0}=10^{\circ}$. However, we sometimes cause an estimation error of these parameters. So the change of jumping height are calculated with respect to the weight perturbation $\Delta M$ and the angler perturbation $\Delta \phi_{\ell 0}$. Figure 17 and 18 show the jumping height with respect to $\Delta M$ and $\Delta \phi_{\ell 0}$. From the dynamical point of view, it is true that the lighter weight and the smaller initial angle yield the higher jumping even though using the circular gear or non-circular gear. However, for the perturbation of these parameters, we obtain the higher jumping by using the non-circular gear than using the circular gear.

\section{Experimental evaluation}

\subsection{Prototype of the jumping robot}

Based on the same specification as in section 3, a jumping robot is prototyped, which is shown in Fig. 19. Figure 19 (a) shows a 3D-CAD model, Fig. 19 (b) shows the configuration of three gears. Gear 2 is a non-circular gear. Link A in Fig. 19 (c) is an additional link to keep the foot link horizontal.

Based on the pitch curve in Fig. 9, a non-circular gear is prototyped as shown in Fig. 20. Unfortunately, the pitch curve in Fig. 9 has an indifferentiable point. Thus a spline interpolation is employed to obtain a smooth curvature. It is $10 \mathrm{~mm}$ thick with 1.5 module, and alloy tool steel (SKD11) is manufactured by wire cut.

\subsection{Jumping experiments}

By using the prototype, the jumping experiments are executed. The results are shown in Fig.21 using the non-circular gear $\left(G_{\min }=1\right)$ in (a) and the circular gear in (b). The jumping motions finish in $0.4 \mathrm{sec}$ and a ratchet mechanism keeps 


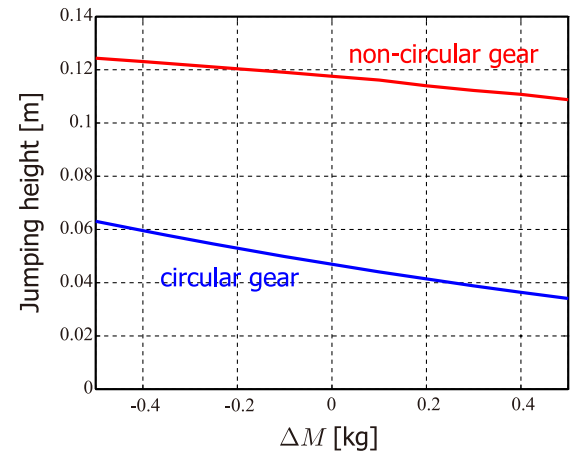

Fig. 17 Change of jumping height with respect to body weight

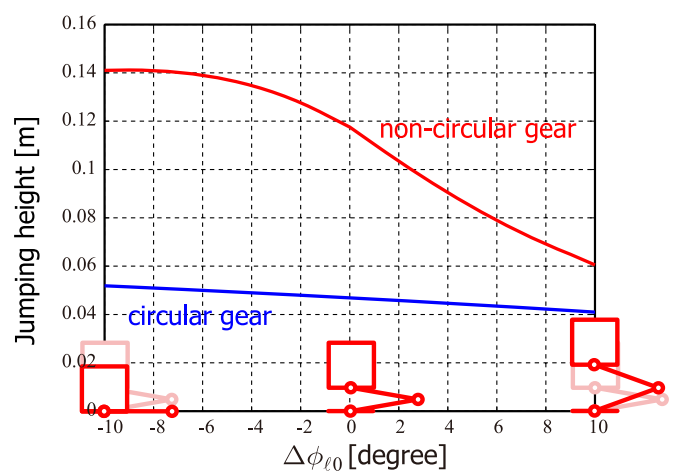

Fig. 18 Change of jumping height with respect to initial posture

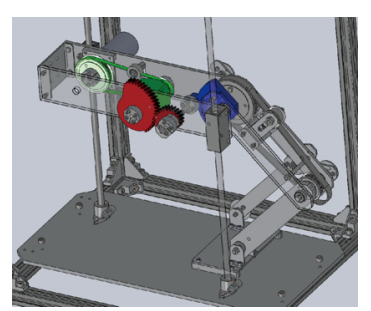

(a) 3D-CAD model

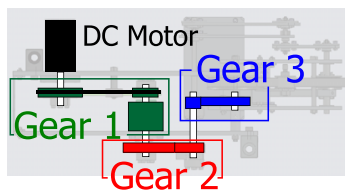

(b) gear configuration

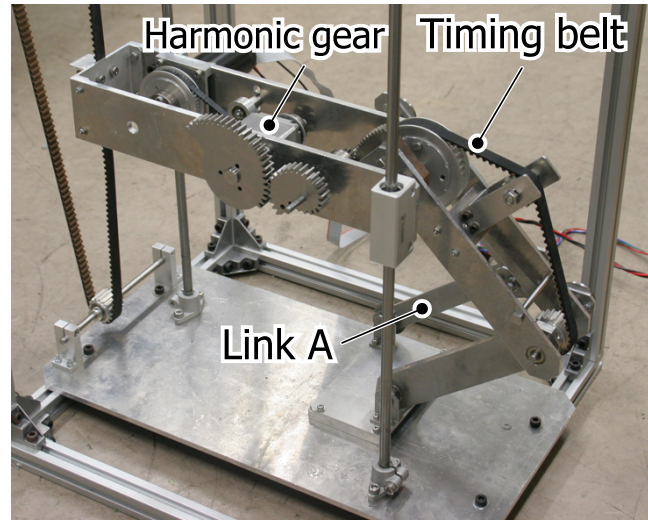

(c) experimental system

Fig. 19 Jumping robot (experimental system)

the jumping height. In figure (a), the jumping height is $0.135 \mathrm{~m}$ using the non-circular gear, and $0.089 \mathrm{~m}$ in (b) using the circular gear. These results show that the higher jumping is realized by using the non-circular gear. Figure 22 shows a comparison of $\dot{y}$ between the experiment and simulation using the non-circular gear. $\dot{y}$ in the experiment is calculated from a measured data of an encoder on the robot joint (time difference). A sampling time of data capture is too short and it contains impulsive noise because of a resolution of the sensor. Because of modeling errors on friction term or robot weight, the time to jump has a little difference, but shows a similar time variation.

As shown in section 3.4, the change of jumping height is measured with respect to perturbations of the initial posture. The non-circular gear is designed with $\phi_{\ell 0}=10^{\circ}$ but it is changed $\phi_{\ell 0}+\Delta \phi_{\ell 0}\left(-1 \leq \Delta \phi_{\ell 0} \leq 5\right)$. The experimental results are shown in Fig. 23. The solid lines show experimental results. The red lines uses the non-circular gear, the blue line uses the circular gear. The dashed lines show the simulation results in Fig. 18. Because of the experimental setup, the range of $\Delta \phi_{\ell 0}$ is much smaller than simulations. Same as the simulation results, by using the non-circular gear, the jumping is higher than circular gear even though a perturbation of the initial posture.

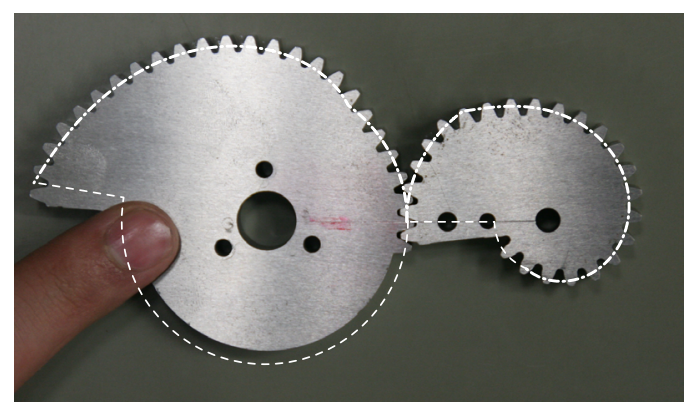

Fig. 20 Prototype of the non-circular gear 

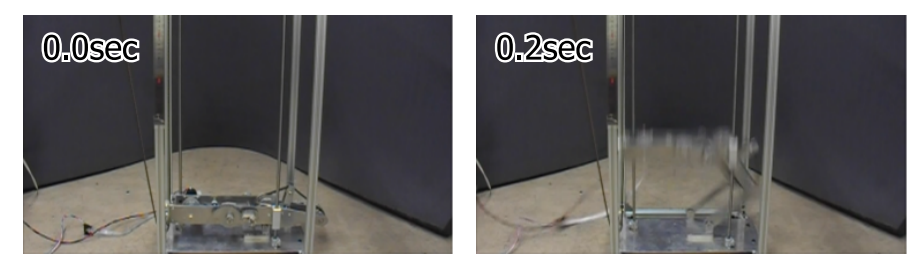

(a) Non-circular gear
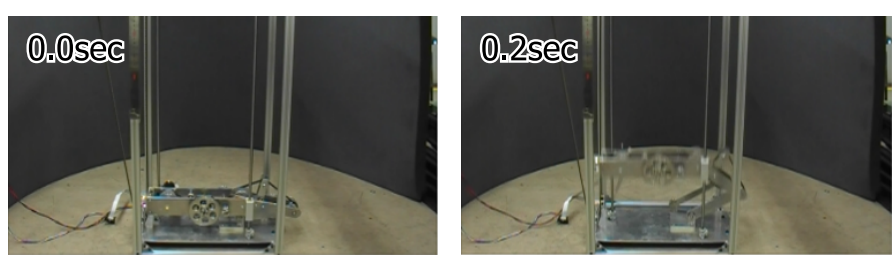

(b) Circular gear
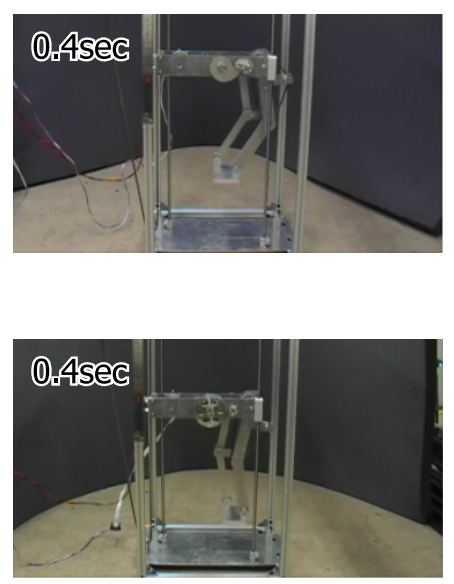

Fig. 21 Experimental results of jumping

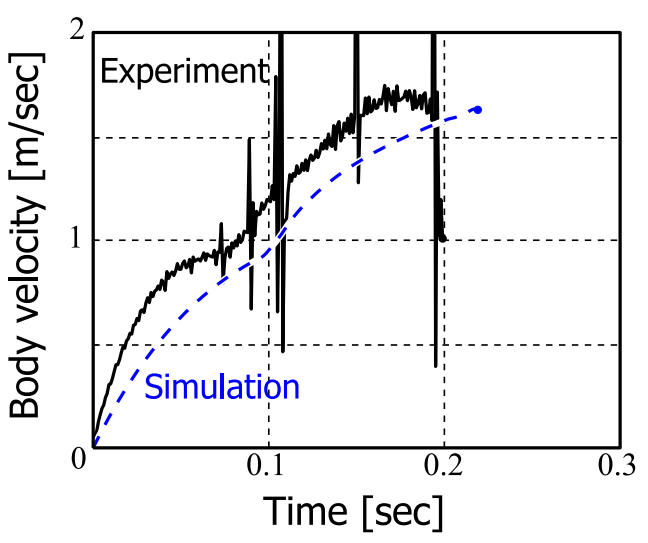

Fig. 22 Comparison between experiment and simulation of $\dot{y}$

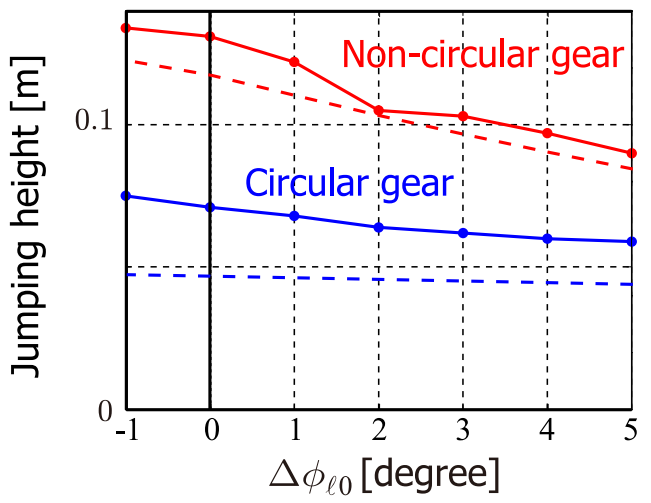

Fig. 23 Change of jumping height with respect to initial perturbation

\section{Conclusions}

In this paper, we focus on a jumping robot, the appropriate gear ratio is introduced and realized by a non-circular gear for the effective use of motor power. A jumping robot is prototyped and the effectiveness of a varying gear ratio is evaluated. The results are summarized as follows.

(1) The appropriate gear ratio that maximizes the ground force is calculated, and its simulation-based design algorithm is proposed.

(2) Based on the obtained gear ratio, a non-circular gear is designed.

( 3 ) Because the proposed method is based on forward dynamical analysis, the results depend on the perturbation of physical parameters of the robot. Thus, the changes of the jumping height with respect to robot weight and initial posture are investigated.

( 4 ) The jumping robot is prototyped with a non-circular gear, and the effectiveness of the proposed method is evaluated.

\section{References}

Curran, S. and Orin, D. E., Evolution of a Jump in an Articulated Leg with Series-Elastic Actuation, Proc. of the 2008 IEEE International Conference on Robotics and Automation (ICRA2008), (2008), pp.352-358.

Hagiwara, T. and Hirose, S., Development of Dual Mode X-Screw : A Novel Load-Sensitive Linear Actuator with a

Wide Transmission Range, Proc. of the 1999 IEEE International Conference on Robotics and Automation, (1999), pp.537-542.

Ishikawa, M., Neki, A., Imura, J. and Hara, S., Energy preserving control of a hopping robot based on hybrid port- 
controlled Hamiltonian modeling, Proc. of 2003 IEEE Conference on Control Applications (CCA2003), Vol.2 (2009), pp.1136-1141.

Katori, H., Design, fabrication and application of non-circular gear, Nikkan Kogyo Shinbun (Business \& Technology Daily News), (2001) (In Japanese).

Kovac, M., Fuchs, M., Guignard, A., Zufferey, J.C. and Floreano, D., A miniature 7g jumping robot, Proc. of the 2008 IEEE International Conference on Robotics and Automation (ICRA2008), (2008), pp.373-378.

Niiyama, R. and Kuniyoshi, Y., Design of a Musculoskeletal Athlete Robot : A Biomechanical Approach, Proc. of 12th International Conference on Climbing and Walking Robots and the Support Technologies for Mobile Machines (CLAWAR 2009), (2009), pp.173-180.

Sakaguchi, K., Sudo, T., Bushida, N., Chiba, Y., Asai, Y. and Kikuchi, K., Wheel-Based Stair-climbing Robot with Hopping Mechanism - Fast Stair-climbing and Soft-landing by Vibration of 2-DOF system -, JSME Journal of Robotics and Mechatronics, Vol.19, No.3, (2007), pp.258-263.

Shimoda, S., Kubota, T. and Nakatani, I., New Mobility System Based on Elastic Energy under Microgravity, Proc. of the 2002 IEEE International Conference on Robotics and Automation (ICRA2002), (2002), pp.2296-2301.

Takaki, K., Sugiyama, K., Takayama, T. and Omata, T., Development of two DOF finger using load-sensitive continously variable transmissions and ultrasonic motors, Advanecd Robotics, Vol.20, No. 8, (2006), pp.897-911.

Tsuda, T., Mochiyama, H. and Fujimoto, H. , A Compact Kick-and-Bounce Mobile Robot powered by Unidirectional Impulse Force Generators, Proc. of the 2009 IEEE/RSJ International Conference on Intelligent Robots and Systems (IROS2009), (2009), pp.3416-3421.

Ugurlu, B. and Kawamura, A., Real-time Running and Jumping Pattern Generation for Bipedal Robots based on ZMP and Euler's Equations, Proc. of the IEEE/RSJ International Conference on Intelligent Robots and Systems (IROS2009), (2009), pp.1100-1105. 